\title{
Why do we need accreditation of nuclear medicine departments?
}

\author{
Amparo García-Burillo • Andrew Hilson • Siroos Mirzaei
}

Published online: 24 July 2012

(C) Springer-Verlag 2012

Nuclear medicine is one of the most dynamic areas of medicine with continual technological innovations and developments of new radiotracers. The International Atomic Energy Agency (IAEA) defines nuclear medicine as a medical specialty that uses techniques with high cost-benefit index to obtain functional and anatomical information, constituting a tool for the detection, staging, treatment, prognosis and monitoring of patients [1]. Nuclear medicine has been an independent European medical specialty since 1988.

The Section of Nuclear Medicine was created in 1990 within the European Union of Medical Specialists (UEMS), the entity that represents the medical specialties inside the European Union (EU) and that defines the basic principles of training in medical specialties in Europe in order to achieve a comparable level of knowledge and to allow the free movement of specialists among the member countries.

The European Board of Nuclear Medicine (EBNM) was established in 1993 with the main objective of ensuring the highest possible levels of quality in the field of nuclear medicine. In 2000 the European Association of Nuclear Medicine (EANM) set up a Task Group on departmental accreditation. In 2003, the UEMS Section and the EBNM merged as the UEMS/EBNM to unify and facilitate their activities and created several committees, including the Committee for Accreditation of Nuclear Medicine Departments (CANMD). This was the EANM Task Group which

\footnotetext{
A. García-Burillo $(\bowtie)$

Hospital Universitari Vall d'Hebron,

Barcelona, Spain

e-mail: amparo.garciaburillo@gmail.com
}
A. Hilson
Royal Free Hospital,
London, UK
S. Mirzaei
Wilhelminenspital,
Vienna, Austria

transferred from the EANM. In practice, the UEMS/EBNM and the CANMD work in cooperation with the EANM.

Technological innovations and advances in the field of new radiopharmaceuticals are very important for the continuous development of the nuclear medicine specialty. Any department of nuclear medicine must assure minimum quality requirements according to its available resources.

Quality! Never has this word been used so frequently. In fact, in recent times there has been a significant reorganization of the concept of quality. Since 1947, the year the International Standards Organization (ISO) was created, there has been increasing emphasis on the quality of products and services. What before had been performed in a routine local way, nowadays attempts are made to globalize the process through standards and a good quality program and well-defined operating procedures, all of which, in our case, are to be applied to a department of nuclear medicine.

Whether a department of nuclear medicine is public or private, it is assumed that it works correctly and assures minimum quality requirements. On this basis, one could ask: "what do I need to get an official accreditation by EANM? - why, in addition to possible certification by ISO and all the daily problems of budget cuts or concern for expenses generated by the cost of new equipment?"

It is possible that many European nuclear medicine doctors have received training in concepts such as total quality, standardization, Deming circles, development of quality policies, objectives and indicators, and that they may now be applying those concepts in their daily work, in an effort to improve patient care and the quality of the services provided by their departments of nuclear medicine, in order to stand out from the other competitors and (why not) to survive in these very competitive times marked by the rampant crisis in all sectors. But it is also true that some other nuclear medicine physicians are unaware of these concepts.

At CANMD we believe that the accreditation of nuclear medicine services is not the mere collection of a diploma to hang on the wall. To have this official accreditation is relevant and provides multiple benefits that outweigh any sacrifice. 
Accreditation means "to give confidence" to all interested parties (institutions, authorities, doctors and patients). It represents an assessment tool that ensures a high level of quality and also gives public recognition and added value to its bearer, which, in many cases, can influence the capacity for innovation and development of the department.

Accreditation ensures correct daily practice, continuous offering of consistent services, permanent uniformity and confidence in the results, availability of technical and human capabilities and international recognition inside the accredited department. It is a way to make society and institutions know that the work is being done efficiently and orderly, in a responsible and competitive way, providing confidence and security to the services provided. Ultimately, the accreditation increases the quality of nuclear medicine services across Europe, which has the added benefit of increasing public awareness and credibility in the specialty and, ultimately, in patient care, which is the most important issue.

On the other hand, accreditation could serve as part of the process of recognition of educational centres in the fields of nuclear medicine (physicians, radiopharmacists, physicists, technologists, etc.) by EANM and UEMS. This means that by accreditation, your department can opt for recognition on the part of the UEMS as a "training centre".

During the accreditation procedure some issues could be discussed with the involved centres, such as: if we can increase the diagnostic sensitivity and performance in patients with suspected pulmonary embolism by carrying out tomographic studies, then why should we confine ourselves to performing planar detections? If we can optimize the dose of radiation, then should we use inadequate doses? If we have PET/CT equipment offering high sensitivity and specificity in the staging of lymphomas..., why should we confine ourselves to studies with ${ }^{67} \mathrm{Ga}$ for the same indication?

In times of financial crisis like those we live in nowadays, obtaining accreditation provides us with an instrument of efficiency and therefore better returns and better process management through a good structure and adequate quality documentation on good processes and good outputs.

This has to be implemented in the EU countries since the Council Directive 97/43/EURATOM on health protection of individuals against the dangers of ionizing radiation in relation to medical exposure was born. This directive on medical exposures to radiation is a requirement of the EU, and one of the main concepts it introduces is that of clinical audits in nuclear medicine [2].

Obtaining accreditation by the CANMD is the basis for the implementation of the Council Directive 97/43/ EURATOM through the processes of ISO, the IAEA or other national external audits. But now is the time to go further and implement the concept of clinical audits, which ultimately is the best tool for measuring the quality of our nuclear medicine services. ISO standards are only based on the structure (what it takes) and processes (what is done). On the other hand, there is a third factor of vital importance in clinical audits: the outcome (what is expected from us), i.e. the real impact of our patient care work as nuclear medicine physicians, which is the ultimate goal of nuclear medicine departments. As a consequence, one of the major goals of the CANMD is to implement clinical audits in order to improve the quality of the results of nuclear medicine explorations.

Currently, nuclear medicine services accredited by the UEMS must have the ISO certification in its latest ISO 9001:2008 form or any other nationally recognized external audit. But ISO standards use a very general concept of quality, which is equally applicable to a bus company, to a screw factory or to a nuclear medicine department.

It is an instrument to ensure existent defined procedures which are reviewed from an administrative point of view. It does not provide any information regarding the actuality of the defined procedures and whether these are in alignment with accepted international standards. Furthermore, it does not survey the existence of competence of the involved physicians, for instance at least one certified physician for nuclear medicine in a given institution as is requested by CANMD. For this reason, the European Atomic Energy Community introduced the new concept of clinical audits within the Council Directive 97/43/EURATOM.

The clinical audit is defined as a tool to improve the quality of patient care, and the experience and results of a department, through a formal review of its systems, methodologies and patient care results in comparison to internationally defined and accepted standards, as well as the implementation of changes based on those results. In other words, the clinical audit compares what we are doing with what we should be doing in order to judge our current clinical practice against national and international standards for each situation in particular.

A symposium conducted in 2003 in Tampere, Finland showed that there was great variation among EU Member States on the way in which clinical audits had been implemented, if any implementation had been carried out at all. It was concluded that there was a clear need for further guidance on clinical audits in order to improve their implementation and for harmonizing approaches among EU Member States. If we succeed in achieving that accreditations and clinical audits are carried out by nuclear medicine professionals themselves, we would have taken a big step.

The key component of a clinical audit is that performance is reviewed (or audited) to ensure that what should be done is being done, and if it is not, it provides a structure that allows the necessary improvements to be made. Let us 
remember that a clinical audit has the goal of continuously improving medical practices, in our case, nuclear medicine practices.

To date, clinical audits have only being implemented in nine EU countries, Finland, Sweden and Germany having been the first countries to establish them.

Obtaining accreditation, far from being something annoying, redundant and cumbersome, can rather become the best tool to manage human and technical resources in the most efficient way, to provide a better quality service; to visualize critical points of the departments of nuclear medicine requiring special attention, sending alerts about underlying problems that have not yet risen to the surface but will do so in the future; to better guide and plan the training of its staff; and above all things, to provide the best care to the patient, which is the ultimate goal of every health care unit.

Obtaining accreditation and the subsequent reaccreditations, and even more when clinical audits are carried out homogeneously throughout Europe, is a powerful administrative and technical warning mechanism that will shed light on dark corners of our nuclear medicine services and, above all, will place highlight indicators to mark the paths that the procedures we do must follow to reach the best possible results, given the resources available to us. And as a great additional benefit, when they are finished, they leave documented recommendations about the changes that could improve our service and reduce expenses, wastage and duplications that we currently have but do not see very clearly, or are not aware of at all.

This accreditation based on carrying out clinical audits, always within the field of quality management systems, will improve the radiation protection of patients, their families and health personnel of the departments of nuclear medicine, by providing the latest indications approved on the handling of radiopharmaceuticals. It will help to discover the existence of incorrect practices and may foresee and avoid accidents and incidents arising from malpractice or lack of preventive measures, as our risk management system and our technical documentation are revised and discussed with other nuclear medicine professionals who will give you points of view and even possibilities for improvement.

\section{References}

1. Nuclear medicine resources manual. Vienna: International Atomic Energy Agency (IAEA); 2006.

2. Mirzaei S, Maffioli L, Hilson A. Clinical audit in nuclear medicine. Eur J Nucl Med Mol Imaging 2011;38:3-4. 